\title{
EL PRINCIPIO DE AUTONOMÍA \\ DE LA PERSONA EN EL DISCURSO \\ MORAL: ANÁLISIS DE UN \\ ARGUMENTO PRAGMÁTICO
}

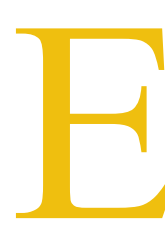

ste trabajo consta de tres partes. En la primera analizo brevemente el concepto de inconsistencia pragmática con el objetivo de estudiar el argumento de C.S. Nino $^{1}$ en el que usa este concepto para justificar el principio de autonomía de la persona a partir del discurso moral. En la segunda parte presentaré y realizaré una reconstrucción de su argumento para demostrar la invalidez formal y proponer algunas variaciones sobre él. Este estudio tiene la finalidad de elucidar el poder justificatorio de un argumento que apele al principio de la contradicción pragmática, y en él llego a la conclusión de que el argumento pragmático no es más útil que la simple deducción de la lógica tradicional ${ }^{2}$, de la cual depende. El poder justificatorio específico de los argumentos pragmáticos dependerá en última instancia del contenido de sus presupuestos. En la tercera parte intentaré demostrar que aún cuando se acepte algún presupuesto mínimo de la discusión moral, el resultado difícilmente será suficiente para fundamentar el principio de autonomía en el sentido tradicional y amplio en que ha de entendérselo.

\section{El concepto de inconsistencia pragmática}

Una inconsistencia pragmática consiste en la contradicción entre un hecho y una proposición. Para la lógica clásica esta contradicción no es posible: la contradicción es una clase particular de proposición y la propiedad de ser contradictorio se predica de las proposiciones de esta clase. Pero la filosofía del lenguaje incursionó en otras áreas considerando también como unidades

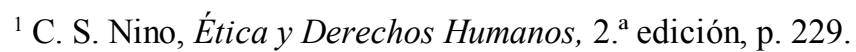

${ }^{2}$ Entiendo por lógica tradicional a la lógica proposicional y de predicados, y no únicamente a la lógica aristotélica clásica. 
de sentido a entidades distintas de las proposiciones, en particular, a los actos de habla. No se desarrolló una lógica que se ocupara de las relaciones formales entre estas entidades ${ }^{3}$. La lógica tradicional se mantiene como el análisis de las relaciones entre entidades que son una parte de algunos actos de habla (la parte o contenido locucionario de los actos de habla declarativos). También hay un desarrollo importante de la lógica deóntica, que investiga la relación entre normas las que, en uno de los sentidos más usuales de «norma» son el contenido locucionario de los actos de habla prescriptivos ${ }^{4}$. La relación lógica entre normas es oscura, particularmente la aplicación y alcance de un principio de no contradicción; pero parece plausible en el ámbito de la moral aceptar que dos normas de algún modo contradictorias no pueden ser a la vez válidas. Sin embargo, aún cuando hay desarrollos de la lógica deóntica que trabajaron con hechos en lugar de proposiciones, no hay casos en que se haya planteado formalmente la relación entre hechos y proposiciones. Esta es la relación que debe clarificarse para elucidar el concepto de contradicción pragmática.

La filosofía práctica encontró en la idea de la contradicción pragmática un método interesante para fundamentar la verdad o al menos la validez de ciertos enunciados no contrastables, a saber, los enunciados éticos. Si se demuestra a alguien que cree en dos proposiciones contradictorias, esta persona, que seguramente asume el principio lógico de no contradicción, se verá comprometida a modificar su opinión sobre alguna de las dos. Si pudiese demostrarse a alguien que hay una contradicción entre algo que hace (un hecho) y algo en lo que cree (una proposición), se verá comprometida (si asume este aún no especificado principio de no contradicción pragmática) a renunciar a este modo de actuar o modificar su creencia. La estrategia usual consiste en seleccionar acciones que no estamos dispuestos a omitir y mostrar que tales acciones son contradictorias con la negación de ciertos enunciados. Esto nos comprometerá a negar esa negación, es decir, a aceptar esos enunciados como verdaderos o válidos.

¿Pero cómo podría un hecho ser contradictorio con un enunciado? Recordemos que los hechos de que hablamos son acciones humanas. Las acciones tienen razones; parte de esas razones son creencias; el contenido de una creencia es o bien una proposición

${ }^{3}$ Salvo quizá para el caso específico de las normas cuando se las considera como hechos.

${ }^{4}$ Este sentido de «norma» que la considera una proposición (en un sentido especial de «proposición») o un enunciado no proposicional, y el anterior que la considera un hecho, son los dos conceptos tradicionales bajo los que se estudia la relación entre normas. 
o bien el contenido de un enunciado no proposicional, como una norma ${ }^{5}$, si lo fuera. La proposición o enunciado puede contradecirse con las proposiciones o enunciados que sean el contenido de otras creencias. Es entre estos elementos del razonamiento pragmático donde se visualizará la contradicción: entre el contenido de diferentes creencias. De este modo la contradicción se dará en última instancia entre enunciados y se descubrirá por aplicación de los principios de la lógica tradicional, o de la lógica deóntica, según el caso. Vemos que la llamada «contradicción pragmática» no es más que la relación que hay entre una acción y una creencia del agente, cuando la acción supone creer en un enunciado contradictorio con el enunciado cuyo contenido es el de la creencia. Para verificar si tal relación existe no será necesario contar con nuevos principios lógicos que den cuenta de relaciones entre hechos y enunciados, sino tan sólo determinar en qué condiciones una acción presupone una creencia.

Para que exista una contradicción pragmática se requiere, por lo tanto, de la presencia de los siguientes elementos: En primer lugar, un hecho que consista en una acción humana o que de algún modo sea capaz de presuponer una creencia de alguien. En segundo lugar, está el hecho de que alguien tiene la creencia presupuesta por aquel hecho. No es claro el concepto de presupuesto, debería determinarse en qué condiciones una acción presupone una creencia, o más bien qué significa presuponer. En lo que sigue consideraremos que la relación de presuposición puede aplicarse a hechos (no me pronunciaré con respecto a la presuposición entre proposiciones u otros enunciados, ni a la presuposición entre creencias) y que al menos parte de su significado es formalizable como una implicación material de modo que lo que está presupuesto es condición necesaria de lo que lo presupone. Diremos que si un hecho presupone una creencia, esto significa (o al menos es parte de su significado) que el hecho de que alguien crea «X》» es condición necesaria de que se dé el hecho en cuestión. Si una proposición presupone otra proposición, esto significará (al menos) que la segunda es condición necesaria de la primera.

El tercer elemento de la contradicción pragmática es la creencia presupuesta por el hecho en cuestión, el enunciado en el cual cree el agente.

${ }^{5}$ En el sentido tradicional de «proposición», ésta es al contenido de un enunciado que puede ser verdadero o falso. Vimos antes (p. 2 y notas 2 y 3 ) que una norma o bien es un hecho, o bien es un enunciado (o su contenido). Cuando se la considera un enunciado, es dudoso que se la pueda considerar verdadera o falsa, se habla en consecuencia de «enunciado no proposicional». 
En cuarto lugar, contamos con otras creencias del agente, que también son enunciados, sean o no proposiciones.

Diremos que hay una contradicción pragmática cuando hay una contradicción, en el sentido tradicional de contradicción, entre el contenido de la creencia que señalamos como tercer elemento, y alguna de las demás creencias del agente.

Para que se entienda claramente la relación de contradicción pragmática cuyo sentido intento elucidar, veamos un ejemplo. Supongamos en primer lugar, como hipótesis que no discutiremos, que el acto de encender la estufa presupone creer que esto calentará el ambiente. Uno podría ciertamente plantearse casos hipotéticos en donde alguien enciende una estufa sin creer que el ambiente se calentará. (Por ejemplo, lo hace al aire libre, y sólo para verificar si la estufa enciende). Pero supongamos a modo de hipótesis que esto no puede ocurrir y que realmente si alguien enciende una estufa, necesariamente cree que se calentará el ambiente. Supongamos ahora que «X» enciende la estufa y por lo tanto cree que el ambiente se calentará. De este modo diremos que el hecho de que «X» encienda la estufa presupone el hecho de que cree que se calentará el ambiente. «X» cree también otras cosas, como que hace frío hoy, que el sol sale todos los días, y que el día tiene veinticuatro horas. Supongamos que también cree que el ambiente en el que está es muy abierto y que no puede calentarse porque hay corrientes de aire que no lo permiten. Si realmente cree esto (como suponemos), entonces esta creencia es contradictoria con la creencia de que se calentará el cuarto, hay una contradicción en el sentido tradicional entre el contenido proposicional de sus dos creencias y, en consecuencia, hay una contradicción pragmática entre el hecho (o acción) de encender la estufa, y la creencia de que no se calentará el ambiente. Si «X» notara la contradicción se vería comprometido a modificar alguna de sus creencias contradictorias, por ejemplo, admitiendo que aunque el cuarto es muy aireado, se calentará al menos algo, y que en consecuencia no es irracional que encienda la estufa. $\mathrm{O}$ bien podría modificar su creencia de que el ambiente se calentará, pero como esta creencia está, por hipótesis, presupuesta en la acción de encender la estufa, « $\mathrm{X}$ » tendrá que omitir esa acción para ser racional, es decir, para evitar la contradicción (pragmática).

En uso del concepto de contradicción pragmática que espero haber elucidado adecuadamente, los filósofos pragmáticos razonan por condiciones de posibilidad: parten de la verificación de un hecho y se preguntan en qué condiciones es posible ese hecho, es decir, qué condiciones son necesarias para que ese hecho se dé. Si el hecho es una acción, y si actuar de cierto modo presupone 
ciertas creencias porque sólo una creencia tal explica la realización de la acción, entonces queda demostrado que el agente tiene esa creencia y, en esas condiciones, el agente sería contradictorio si afirmara algún otro enunciado que la contradijese. Si se parte de una acción generalizada, como la actividad de discutir moralmente, entonces el conjunto de agentes a los que alcanzará el compromiso con la creencia presupuesta será más amplio. De este modo los filósofos morales vieron en la idea de contradicción pragmática un mecanismo útil para la justificación moral objetiva (en un sentido de «objetiva» que podría especificarse de distintas maneras).

\section{1. El argumento de Nino}

C. Nino define a la autonomía como «la libre elección individual de planes de vida y la adopción de ideales de excelencia humana», y defiende un principio de autonomía que consta de dos partes. La primera afirma el valor de la autonomía. La segunda proscribe ciertas clases de interferencia en el ejercicio de la autonomía. Discutiremos aquí el argumento que da para la defensa de la primera parte del principio, es decir, el que afirma el valor de la autonomía persona. No discutiremos la justificación de la segunda parte pero asumamos, como lo admite Nino, que depende de la plausibilidad del argumento que vamos a discutir.

Lo presentaré de un modo levemente distinto al que usó $\mathrm{Nino}^{6}$, dado que en su formulación no hay estrictamente un caso de contradicción entre los contenidos de dos afirmaciones, es decir, entre dos enunciados (o sus contenidos).

La primera parte del argumento es la presentación de la acción que será uno de los términos de la contradicción. La segunda será la explicitación de la creencia presupuesta en la acción. La tercera consistirá en la creencia contradictoria (en el sentido tradicional) con la anterior y que constituirá el segundo término de la contradicción pragmática. El argumento sirve para demostrar al actor la falsedad de la segunda creencia mencionada. Éste, por aplicación de la regla que en la lógica se llamó «reducción al absurdo» inferirá la falsedad de una de las dos creencias contradictorias, en particular la segunda, dado que su acción lo compromete a conservar la primera. La regla podría formularse así: si dado un supuesto o hipótesis, su adición al sistema origina una contradicción (o si agregado un supuesto al sistema se infiere de

${ }^{6}$ Ob. cit., pp. 232-233. 
éste una contradicción), entonces debe inferirse la negación del supuesto ${ }^{7}$.

Presentaré el argumento lógico realizado a partir del contenido de las dos creencias. La conclusión será un enunciado que formará parte del sistema de creencias del agente. La conclusión de lo que podríamos llamar «argumento pragmático» será el compromiso del agente con la conclusión del argumento lógico tradicional incluido en aquél.

Argumento A

1. El agente «X» participa en una discusión moral.

2. Creencia presupuesta: Es deseable que la gente actúe sobre la base de libre adopción de principios morales que, luego de suficiente reflexión, juzga válidos.

3. Creencia propuesta en la discusión como válida: No es deseable que actuemos sobre la base de la libre adopción de principios morales autorreferentes que no son válidos, aunque los consideremos tales. (En el argumento presentado por Nino el agente al presentar este principio, da un fundamento diciendo que se infiere de algún modo no formal a partir de la creencia enunciada en 2 , pero que se aluda a este fundamento no tiene efectos en la corrección del argumento).

4. Conjunción de 2 y 3 . Enunciado contradictorio.

5. Inferencia de la lógica tradicional a partir de los contenidos de las creencias 2 y 3 , y de su conjunción 4, por aplicación de la regla de reducción al absurdo: Es deseable que actuemos sobre la base de la libre adopción de principios morales autorreferentes que consideramos válidos, aunque no lo sean.

6. Conclusión de la lógica pragmática: «X» está comprometido a afirmar 5.

${ }^{7}$ Puede darse otras formulaciones de reglas de inferencia útiles e igualmente justificables intuitivamente. Alguna otra podría tener las características de considerar a ambas creencias como supuestos y permitir la inferencia de la disyunción de sus negaciones, dejando libertad al agente para seleccionar cuál de sus creencias mantendrá en el sistema y cuál descartará como un supuesto que resultó inválido o falso. Creo que esto no resultaría una ventaja dado que considerar a la creencia presupuesta en la acción como perteneciente al sistema, da cuenta del hecho de que no existe la opción de negar este enunciado por lo que necesariamente la creencia a descartar será la segunda. Una inferencia que tomara a ambos enunciados como supuestos deberá incorporar posteriormente al sistema la afirmación del enunciado presupuesto usando de premisa esta afirmación para inferir la negación del otro. Los resultados serán idénticos. 
En esta reconstrucción del argumento de Nino, el único elemento que podría discutirse con respecto a la fidelidad con el argumento original es, creo, el paso número 5. Esto es así porque Nino no expresa en estos términos la conclusión sino que afirma que «es inconsistente la intención de quien participa lealmente en el discurso moral para negar el principio de autonomía en la elección de modelos de excelencia humana» ${ }^{8}$. Si no puede negarlo porque sería inconsistente, entonces Nino supone que está comprometido a afirmarlo; su afirmación será, presumiblemente, la negación del enunciado en el que el participante de la discusión niega el principio de autonomía; esta doble negación será lógicamente equivalente al principio de autonomía mismo. En suma, una interpretación plausible del argumento que estudiamos, supone que 3 es la negación del principio de autonomía, y que 5 se infiere de 3 por doble negación, constituyendo el principio de autonomía. Pero la definición que da Nino del principio de autonomía, y de la autonomía, no están expresados en términos similares a los que usa en su argumento y que reprodujimos en el paso 3 . Comparar:

3. (No es deseable) «que actuemos sobre la base de la libre adopción de principios morales autorreferentes que no son válidos aunque los consideremos tales».

Con la definición del principio de autonomía según el cual: (es valiosa) «la libre elección individual de planes de vida y la adopción de ideales de excelencia humana...»».

De este modo la conclusión 5 no puede consistir en el principio de autonomía expresado en los términos de Nino. Debe ser, en cambio, la negación de 3. Será necesario un análisis semántico posterior para determinar si el enunciado que puede inferirse negando 2 es equivalente al principio de autonomía.

Por otra parte, aún cuando nos hayamos puesto de acuerdo en que 5 debe estar expresado en términos similares a los de 3, usando las mismas propiedades que en éste, y en que debe ser su negación, tendremos que acordar asimismo cuál es la negación de 3 . Una razón por la cual seleccionamos arriba la conclusión (5) como lo hicimos es que la forma gramatical del enunciado es similar a 3, pero tiene carácter negativo; esto podría llevar a suponer que los dos enunciados son uno la negación del otro. La segunda razón es que la conclusión que propusimos tiene carácter general, y ha de tener tal carácter si de algún modo debe ser equivalente al principio de autonomía, o si ha de implicarlo.

Así presentado el argumento que discutimos, analizaremos su

${ }^{8}$ Nino, C. S., Ob. cit., p. 233.

${ }^{9}$ Nino, C. S., Ob. cit., p. 204. 
validez. Una vez demostrado que, así como lo reconstruimos, es inválido, propondremos algunas variaciones válidas y su poder, así como el de esta clase de argumentos, para inferir una conclusión que pueda ser fundamento del principio de autonomía de la persona. Por último, haremos el análisis semántico que muestre en qué medida el principio está implicado.

\subsection{Análisis formal del argumento}

El argumento pragmático, como vimos consta de:

- Un primer paso acerca de un hecho, en particular, una acción.

- Un paso (al menos) acerca de las creencias presupuestas por ese hecho.

- Un paso (al menos) acerca de hipótesis o supuestos adicionales.

- Uno o más pasos intermedios que permitan hallar una contradicción entre las creencias presupuestas y las supuestas como hipótesis.

- Una conclusión intermedia en donde se infiere la negación de algún supuesto del que dependa esa contradicción.

- Un paso final en el que se afirma la existencia de un compromiso del agente con el contenido del paso anterior.

Los pasos que siguen al primero, referente al hecho en cuestión, y que anteceden al último, referente al compromiso del agente que participa en el hecho, son proposiciones, y la inferencia de una contradicción, así como los demás pasos que trabajan con proposiciones, se manejan exclusivamente con el uso de la lógica tradicional y es por este método que alcanza la conclusión intermedia que será el contenido de la conclusión final. La validez del argumento pragmático en general depende de la validez del argumento de la lógica tradicional que forma parte de aquél. Analizaremos por tanto de aquí en adelante la validez de esta parte del argumento, que abarca los pasos 2 a 5 de la formulación que hicimos.

Veamos entonces la forma lógica de los enunciados que componen esta parte del argumento.

Si pensamos en la forma lógica del enunciado 3, veremos que tiene carácter general. Afirma algo (que no son deseables) de todos los casos de acción en base a la libre adopción de cualquier principio moral autorreferente que no sea válido aunque lo consideremos tal. La negación de un enunciado general es un enun- 
ciado particular que afirma que hay al menos algo que no tiene la propiedad (o las propiedades) en cuestión. En nuestro caso: «hay alguien que puede actuar (no es indeseable o disvalioso que actúe) en base a un principio moral autorreferente que considera válido aunque no lo es».

Podría pensarse, aferrándonos a la forma gramatical, que 3 no es general, sino que más bien habla de una clase de cosas, de un incontable como es la acción (cierta clase de acción), la voluntad, el agua, etc. Que nos referimos a ese elemento general y afirmamos que posee ciertas propiedades. El agua es líquida a cierta temperatura y presión, o no lo es, y la negación de que el agua es líquida es que no lo es, y no que hay ciertas clases de agua que no lo son.

Pero este argumento es insuficiente, porque la razón por la que pensamos que se puede hablar de los incontables como de particulares afirmando o negando propiedades de un elemento (el agua) que también podría definirse como una clase de cosas (las que son agua), es que pensamos que esta clase de cosas es uniforme. Pensamos que el agua siempre es líquida en ciertas condiciones, o no lo es. Si no supusiéramos esta uniformidad no podríamos inferir de nuestro conocimiento de que es falso que el agua de mar es dulce, que no lo es. Deberíamos decir que al menos hay casos en que no lo es.

Supongamos este caso similar al nuestro: «El agua de mar se ve azul». Este enunciado afirma que el agua de mar siempre se ve azul. Su negación expresaría que al menos a veces (por ejemplo, en el caso del Mar Negro) no se la ve azul, y no que «el agua de mar nunca se ve azul», que sería el significado de la negación aparente: «El agua de mar no se ve azul». No hay por qué suponer la uniformidad del agua, es decir, que el agua (toda) tiene siempre las mismas propiedades. Del mismo modo, no hay por qué suponer que la actuación sobre la base de ciertos principios (elegidos libremente, etc.) tiene siempre las mismas propiedades. Una asunción como ésta debería ser debidamente fundamentada e incorporada al argumento.

Recordemos que en el argumento, 3 funciona como un supuesto que al originar una contradicción en 4 permite inferir 5 , por medio de la negación del supuesto 3 . Si percibimos que el enunciado se entiende claramente como una alusión a todos los casos que tienen ciertas características, se verá también que su negación es un enunciado particular, y que no coincide con la conclusión 5. La negación de que no es deseable que actuemos sobre la base de la libre adopción de principios morales autorreferentes que no sean válidos aunque los consideremos tales, es que hay al menos algún caso en el cual es deseable que alguien actúe según algún 
principio moral, autorreferente, libremente adoptado, no válido (creyéndolo válido).

En consecuencia, vemos que el argumento no es válido, dado que el paso 5, consistente en la negación de 3 (y posterior eliminación de la doble negación resultante), da como resultado el anunciado particular recién expresado ${ }^{10}$. No parece plausible que

${ }^{10}$ Formalizaré el argumento de la lógica tradicional del cual depende, como vimos, la validez del argumento de la lógica pragmática que analizamos. Se verán así más claramente la forma lógica, la inferencia que se puede hacer válidamente, y el carácter particular de la conclusión permitida.

Interpretación de las constantes que representan propiedades:

$\mathrm{Mxy}=$ Ser «y» un principio moral libremente adoptado por « $\mathrm{x} »$.

Dxy $=$ Ser deseable que $\langle\mathrm{X} »$ actúe según $« \mathrm{y} »$.

Ty $=$ Ser «y» válido.

Ay $=$ Ser $\langle y »$ autorreferente.

Para simplificar el argumento excluiré la propiedad de los principios morales en cuestión de ser considerados válidos por el agente. Esta propiedad la tomaremos como incluida en la idea de libre aceptación de un principio moral: aceptar un principio moral como tal incluye considerarlo válido.
1. $(\mathrm{x})(\mathrm{y})(\mathrm{Mxy}->$ Dxy)
2. (xc)(y) [(Mxy.Ay.-Ty) -> -Dxy]
3. (x)(y) (Mxy -> Dxy).[Mxy.Ay.-Ty) -> -Dxy]
4. (Ex)(Ey) (Mxy.Ay.-Ty)
5. (Ex)(Ey) (Dxy.-Dxy)
6. (Ex)(Ey) (Mxy.Ay-Ty.Dxy) presup. de la acción supuesto

Conj. 1 y 2

Supuesto

Inf. de 3 y 4

De 2 y 5 xRed. Abs.

La conclusión descansa en dos pasos. En 1, que es el presupuesto de la acción presentada en el argumento y que no tenemos opción de negar. Puede ser tomado como premisa. Y en 4, que es el supuesto débil de que hay al menos alguien que acepta libremente y procede según un principio moral autorreferente inválido. Este supuesto es necesario para que pueda extraerse de 3 una contradicción. No creo que haya grandes dificultades en admitirlo, dado que su negación significaría afirmar que nadie procede según ningún principio moral, autorreferente, inválido, libremente aceptado. Si además admitiéramos que estos enunciados existenciales no se refieren a una existencia actual, sino a cualquier existencia posible, entonces las condiciones de verdad de 4 se volverían aún más débiles, exigiéndose sólo la posibilidad lógica de que se dé una situación como la expresada en 4.

La suposición de 4 soluciona una conocida paradoja del condicional, según la cual todo condicional es verdadero cuando su antecedente es falso. Esta paradoja tiene la consecuencia de que un enunciado general que predica de todas las cosas que tienen la propiedad $\mathrm{F}$, que también tienen la propiedad $G$, sería verdadero si no existiera ninguna cosa que tuviese F. Pero esto parece contraintuitivo y una solución plausible es afirmar que siempre que formulamos un enunciado de esa clase estamos diciendo implícitamente que hay (en algún sentido de «haber» al menos alguna cosa que tiene la propiedad F señalada en el antecedente. Si admitimos esto admitiremos también que no es contraintuitivo interpretar al enunciado 2 como una afirmación implícita del enunciado 4 . Es porque consideramos implícita 4 que nos parece (y así se infiere del argumento) que 1 y 2 son contradictorias, y que de su conjunción, 3 , se infiere una contradicción. 
éste sea equivalente al principio de autonomía o que pueda implicarlo de algún modo.

\subsection{Algunas variaciones sobre el argumento}

Sigo basándome en el argumento anterior, pasos 2 a 5 .

La alternativa más obvia es modificar el supuesto 2, para que efectivamente sea la negación del principio de autonomía o de la conclusión de la que esperamos inferirlo. La primera condición para que la conclusión sea apta para esto es que sea general. Su negación ha de ser un enunciado particular como el siguiente:

3'. Hay casos en los que alguna persona actúa sobre la base de algún principio moral autorreferente que no es válido, en los cuales no es deseable que el agente actúe según esos principios.

Si reemplazamos en el argumento original 3 por 3', el segundo paso que resulta distinto es el número 4, que consiste en la conjunción de los dos pasos anteriores y que debe ser o implicar una contradicción para que la regla de la reducción al absurdo permita inferir la negación del supuesto (3’).

Si tomáramos textualmente el enunciado 2 nos encontraríamos con que predica que son deseables ciertas conductas que tienen las propiedades de ser realizadas según principios morales libremente adoptados y de ser juzgados válidos luego de suficiente reflexión. El enunciado 3', en cambio, predica algo de (al menos) una conducta individual con ciertas propiedades sin tomar en cuenta la propiedad de que los principios a los que se refiere sean juzgados válidos luego de suficiente reflexión. Por lo tanto, podría darse el caso de que los casos de conductas no deseables a que refiere este enunciado fuesen aquellos en que no se dio el requisito de la reflexión suficiente, casos no alcanzados por el primer enunciado. Esta alternativa muestra que los enunciados, así formulados, no son contradictorios.

Podríamos solucionar esa dificultad incluyendo la propiedad de la reflexión suficiente tanto en 3' como en la conclusión, la que será en consecuencia un poco más restringida. Por razones de simplicidad lo que haré será excluirla de 2. Por causas similares excluiré el requisito de que los principios morales sean considerados válidos por el agente, considerándolo incluido en la adopción de un principio moral como tal. El nuevo argumento, presentado un poco más formalmente que el primero, queda conformado como sigue. Únicamente presento la parte que trabaja con la lógica tradicional, dado que, como vimos, de la validez de ésta depende la del argumento total. Altero también la numeración 
anterior.

Argumento $\mathrm{B}^{11}$

1. Es deseable que la gente actúe en base a la libre adopción de principios morales.

2. Hay al menos un caso de acción según la libre adopción de algún principio moral autorreferente inválido, en el que no es deseable que el agente actúe según ese principio.

3. Conjunción de 1 y 2. (Enunciado contradictorio).

4. Es deseable que la gente actúe según la libre adopción de principios morales, autorreferentes (aunque sean) inválidos. (Enunciado equivalente a la negación directa de 2: «No hay ningún caso de acción realizada según (al menos un) principio moral autorreferente, libremente adoptado, inválido, en el que no sea deseable que el agente actúe según ese principio»).

Este argumento es válido. Mi objeción no es contra la corrección de sus pasos sino contra la practicidad del recurso del argumento pragmático de proponer una hipótesis que finalmente resulta contradictoria con alguna creencia presupuesta, para negar la hipótesis e inferir así la conclusión deseada. El método no es práctico porque si analizamos la conclusión veremos que, a pesar de su carácter general, es demasiado restringida; su extensión es un subconjunto de la extensión del paso número 1, la creencia presupuesta en la acción de discutir moralmente, y que aquí funciona como premisa. Los principios morales, autorreferentes, inválidos, son una subclase de los principios morales en general a los que hace referencia 1 , de modo que 4 pudo haberse inferido directamente de él. Si 4 fuese equivalente al principio de autonomía, podría justificárselo directamente a partir del presupuesto. Pero 4 es más restringido que 1, de modo que podría justificarse un principio

${ }^{11}$ Interpretación:

Mxy $=$ Ser «y» un principio moral libremente adoptado por « $\langle\mathrm{x}\rangle$.

Dxy $=$ Ser deseable que «x» actúe según «y»».

Ty $=$ Ser «y» válido.

Ay $=$ Ser $\langle y »$ autorreferente.

Argumento B

1. (x)(y) (Mxy -> Dxy)

premisa

2. (Ex)(Ey) (Mxy.Ay.-Ty.-Dxy)

supuesto

3. (x)(y)(Mxy -> Dxy).(Ex)(Ey)(Mxy.Ay.-Ty.-Dxy)Dxy).

Conj. 1 y 2

3'. (Ex)(Ey) [(Mxy -> Dxy). -(Mxy -> Dxy)]

Inf. de 3

4. (x)(y) [(Mxy.Ay.-Ty) -> Dxy]

Inf. de 2 y 3

por Red. Abs. 
más general infiriendo directamente de 1 , por identidad, el contenido de éste. 
Para hacer la conclusión más amplia, podríamos modificar 2 constituyéndola exactamente como la negación de 1, de modo que la conjunción de 1 y 2 no sólo fuese contradictoria (implicara una contradicción) sino que fuera en sí misma una contradicción. El resultado obvio sería que la conclusión coincidiera con 1 . Nuevamente hubiese sido más fácil inferirla a partir de 1 , por identidad.

Por último podríamos intentar modificar 2 para que su negación fuese más amplia que 1, y así la conclusión abarcase más casos que el presupuesto. Supongamos, por ejemplo, que queremos arribar al resultado de que es deseable que actuemos según la libre adopción de principios de conducta, excluyendo la propiedad de que sean principios morales. En este caso 2 expresará que hay al menos un caso de acción según la libre adopción de un principio libremente adoptado, en donde no es valioso que el agente actúe según éste. Pero este enunciado no es contradictorio con 1, porque 1 no afirma que sean valiosos todos los casos referentes a principios libremente elegidos sino sólo los referentes a principios morales, de modo que entre aquellos que no incluye podría estar el caso expresado en 2. Así, la contradicción no se formará y no podremos deducir la negación de 2.

En suma, el argumento no permite inferir más que lo incluido en los presupuestos o premisas del argumento. Esto no es extraño, dado que es lo que ocurre en la lógica tradicional y que estamos trabajando con un argumento de esta clase.

La utilidad del argumento pragmático dependerá, en última instancia, del contenido de los presupuestos de la acción o hecho de que parta. Nuestro trabajo consistirá de aquí en adelante, en el estudio de los presupuestos del discurso moral de que habla Nino y que incorpora (o podría incorporar) a su argumento. Analizaré su relación con el principio de autonomía y su aptitud para justificarlo.

El último paso del análisis debería ser una investigación del hecho mismo de la discusión moral para determinar cuáles son sus presupuestos. Si implican el principio de autonomía o, en definitiva, algún principio moral, o de conducta. Esta investigación no puedo encararla en el ámbito de este artículo. Daré sólo algunos argumentos en favor de la tesis de que el principio de autonomía no está presupuesto en el discurso moral y de que en consecuencia, por este método, no puede ser justificado. 


\section{El presupuesto mínimo del discurso moral}

El argumento que da C. Nino en favor del principio de autonomía de la persona está fundamentado en un presupuesto del discurso moral que él considera un presupuesto mínimo. Esto significa que es la afirmación más restringida a que estamos comprometidos al realizar la acción de participar en el discurso, independientemente de nuestro compromiso con otras creencias que pudieran estar presupuestas y con los principios y enunciados que implicasen. Este presupuesto mínimo expresa: «Es deseable que la gente determine su conducta sólo por la libre adopción de los principios morales que, luego de suficiente reflexión y deliberación, juzgue válidos» ${ }^{12}$.

La cuestión que intentaremos clarificar aquí es si este enunciado contiene de algún modo al principio de autonomía de la persona, dado que vimos en los parágrafos anteriores que la plausibilidad de validar tal principio por medio del argumento citado depende de que pueda inferírselo de los presupuestos del discurso.

En primer lugar destaquemos que esta formulación es más fuerte que la que figura en el argumento que veníamos analizando, dado que habla de la determinación de la conducta «sólo por la libre adopción...», de modo que incluye no únicamente la deseabilidad de ciertas formas de determinar la conducta, sino que agrega que las demás formas de determinarla no son deseables. Este contenido sería muy difícil de justificar, y además más extenso de lo que Nino parece necesitar en su argumentación, como lo prueba el hecho de que en adelante menciona el presupuesto en su forma más restringida, hablando sólo de la deseabilidad de las conductas en cuestión. En esta forma es como lo manejaremos de aquí en más, que es coincidente con el presupuesto usado en el argumento de Nino y en las variaciones que hicimos sobre él.

En segundo lugar recordemos que el principio de autonomía consta de dos partes o aspectos, el primero consistente en valorar positivamente la autonomía o libertad de elegir y materializar libremente planes de vida e ideales de excelencia humana, el segundo consistente en vedar al Estado y a los individuos interferir en el ejercicio de esa autonomía. La fundamentación del segundo aspecto depende de la del primero y es éste el que analizamos aquí.

Nino admite, en cuanto al primer aspecto, que el valor del ejercicio de la autonomía no es un valor en sí mismo, sino que está subordinado prácticamente al valor de la autonomía como libre

${ }^{12}$ Ob. cit., p. 230. 
elección de principios. El ejercicio de la autonomía es una condición de posibilidad de la autonomía misma y el valor de la autonomía se extiende a sus condiciones de posibilidad. Por eso resulta extraño que en la argumentación posterior intente fundamentar el valor del ejercicio de la autonomía, es decir, la realización de las acciones que estén de acuerdo a principios libremente elegidos, y no el valor de la autonomía misma. Las consecuencias de esta decisión no vuelven inútil la tentativa, dado que parece intuitivamente aceptable asumir que si es valioso o deseable actuar según ciertos principios libremente elegidos (ejercicio de la autonomía), también lo será elegir libremente esos principios (autonomía). El principio o regla implícita es que son valiosas las condiciones de posibilidad de los hechos valiosos. Pero si bien este mecanismo nos permite inferir el valor de la autonomía, no muestra el nivel de prioridad que Nino pretende que tiene el valor de la autonomía sobre el de su ejercicio. La prioridad más bien aparece invertida.

Por eso creo que es un error de Nino construir el presupuesto mínimo del discurso moral sobre el valor o deseabilidad del ejercicio de la autonomía. Sólo si adoptáramos como valor primario el de la autonomía podríamos inferir como valor secundario el de su ejercicio, en la medida en que fuese necesario asegurar un cierto grado de ejercicio de los principios de conducta a elegir para permitir esa elección (nadie elegiría un principio de acción que supiese que no podrá usar). El valor del ejercicio de la autonomía incluye, como condición de posibilidad, el de la autonomía misma ${ }^{13}$.

Notemos que en el argumento de Nino y en las variaciones que propusimos, la forma lógica no se modificaría si reemplazáramos el concepto de deseabilidad de la conducta realizada según ciertos principios morales, por el de deseabilidad de la libre elección de

${ }^{13}$ Una vez terminado este trabajo, y gracias a las observaciones y respuesta del Dr. Carlos Nino, noté un error mío en este punto, donde hablé de una diferencia entre el valor de la autonomía y el valor de su ejercicio, y la prioridad entre ellos. La distinción a la que pretendí hacer referencia es la que Nino hace entre la libre elección de planes de vida e ideales de excelencia humana, y la realización de esos planes de vida. Ambos son aspectos de la autonomía que consiste en la elección y materialización de esos planes de vida e ideales.

Mi objeción debe leerse entonces como dirigida al argumento de Nino en cuanto se refiere al valor de que la gente actúe según ciertos principios libremente elegidos, y no al valor de la elección de esos principios. Para que el valor del ejercicio de esos principios dependa de la medida en que tal ejercicio sea condición de posibilidad de la elección, el argumento debió haber versado sobre el aspecto de la autonomía relativo a la elección de principios. Si, como hace Nino, se parte del ejercicio de aquellos principios el orden de prioridad aparece invertido. 
esos principios, de modo que con el nuevo presupuesto podría construirse argumentos similares a los estudiados, siendo aplicable el análisis hecho sobre aquéllos. Sea cual fuere el presupuesto mínimo que consideremos implícito en la discusión moral, se refiere a la elección o al ejercicio de ciertos principios, debemos cuestionarnos algo que en los últimos párrafos asumíamos, a saber, si la «libre elección (o ejercicio) de principios morales (que juzgamos válidos luego de suficiente reflexión)», términos usados en los argumentos anteriores, es equivalente a, o implica, «la libre elección (y materialización) de planes de vida y la adopción de ideales de excelencia humana». Estos últimos son, recordemos, los términos en que Nino define el principio de autonomía de la persona.

El Dr. Nino considera que el concepto más amplio entre los que nos ocupan es el de principios morales en general, una subclase de principios morales es el conjunto de las pautas de la moral autorreferente que determinan los planes de vida de las personas ${ }^{14}$, un tercer subconjunto es el de los ideales de excelencia humana que están presupuestos por aquellos planes de vida ${ }^{15}$. De este modo, el valor de la adopción de ideales de excelencia humana estará implicada ${ }^{16}$ por el valor de la libre elección de los planes de vida que presupongan a esos ideales, a su vez la elección de planes de vida será siempre una libre elección de principios morales autorreferentes. Si se prueba que es valiosa la libre elección (o materialización) de principios morales autorreferentes se habrá

${ }^{14}$ Ob. cit., p. 230.

${ }^{15}$ Ob. cit., p. 229.

${ }^{16}$ No es claro qué pueda significar que un plan de vida presupone algún ideal de excelencia humana. Aparentemente no es un caso de presuposición entre hechos, ya que un plan de vida y un ideal de excelencia no son hechos, sino conceptos. Quizá podría pensarse que la elección de un plan de vida es un hecho que presupone el hecho de la adopción de un ideal de excelencia humana. Esto podría deberse a que elegir un plan de vida consiste, entre otras cosas, en adoptar un cierto ideal de excelencia. No intentaré aclarar este punto. Supondremos que la relación de la que habla Nino es de una clase tal que si se demuestra el valor moral de lo que está alcanzado por el primer concepto, se demostrará el valor moral de lo alcanzado por el segundo al que el primero presupone. Para esto no sería suficiente con demostrar que el concepto presupuesto es condición necesaria del otro, dado que no tiene por qué ser verdadero que si algo es valioso o deseable, lo será también cualquier condición materialmente necesaria de aquel (como una consecuencia material, que bien podría ser moralmente indiferente). Pero admitiremos que si algo es, no sólo condición necesaria, sino también condición de posibilidad de otra cosa «X», entonces el valor de «X $\mathrm{X}\rangle$ se extenderá también a aquello que sea su condición de posibilidad. Repetimos que el ser condición necesaria es parte de la definición de ser condición de posibilidad, pero no agota necesariamente su significado. 
probado el valor de la subclase de principios morales a que hace referencia el principio de autonomía.

$\mathrm{Si}$ se aceptan las categorías anteriores, entonces deberemos admitir que efectivamente el principio de autonomía está implicado en el presupuesto mínimo del discurso moral, en cualquiera de sus dos formulaciones, aunque vimos que si escogemos la que hace referencia al ejercicio de acciones se inferirá un error en la prioridad de los aspectos de la elección y la materialización de planes de vida.

Mi objeción aquí es justamente en contra de la aceptación de estas categorías. En primer lugar, restringen demasiado el principio de autonomía. No en el sentido de que se refiere solamente a principios morales autorreferentes, dado que parece aceptable sostener que sólo es valioso escoger un plan de vida cuando es un plan de la vida propia, al menos en el contexto liberal en el que se maneja la teoría que estudiamos. Creo que restringen el principio de autonomía porque según estas categorías sólo se considerará valiosa la elección de pautas de conducta cuando éstas sean principios morales. Sin embargo, en el sentido común de «plan de vida», las razones por las que decidimos vivir de un modo u otro, elegimos una profesión o con quién vivir, no son razones morales. Son más bien razones acerca de lo que queremos, de lo que sentimos importante. No de lo que sentimos bueno, debido, obligatorio. Estas ideas son discutibles, pero me parece claro que un plan de vida, al menos en gran parte, no es un conjunto de principios morales. Y que si a partir de un presupuesto que afirma el valor de la libre elección de principios morales, pretendemos inferir el valor de los planes de vida que hacemos día a día, por razones prudenciales, tal inferencia será imposible. Si, en cambio, restringimos el concepto de plan de vida, para considerar incluidos sólo los principios morales que lo compongan, entonces no habremos conseguido justificar el principio liberal de que es valioso que la gente elija libremente su plan de vida, y decida qué hacer con su tiempo, sus recursos, sus capacidades, al menos en los casos en que esté claro que no debe destinarlos a cumplir obligaciones morales.

Si la libre elección de planes de vida en este sentido amplio no puede considerarse valiosa en base al presupuesto que usamos, esto de ningún modo significa que sea disvaliosa. Bien podría ser moralmente indiferente que alguien eligiera libremente sus metas. La libertad sería deseable sólo en el sentido de que la gente la desea. Esto no implicaría el disvalor de la libertad. Aclaro este punto por si alguien pudiera sentirse tentado a fundamentar el valor de un principio de autonomía amplio en el rechazo de un 
principio contraintuitivo como el citado. La autonomía en general no es disvaliosa. Esto lo sabemos porque probamos que al menos a veces es valiosa, a saber, los casos en que se trata de la libre elección de principios morales autorreferentes. Pero que no sea disvaliosa no significa que sea valiosa. Hay casos en que lo es, y casos, los no abarcados por nuestro presupuesto mínimo del discurso moral (casos de principios no-morales), en que no lo sabemos.

La alternativa más obvia para intentar una ampliación del principio de autonomía es ampliar el presupuesto mínimo del discurso moral, para que lo implique. Deberemos sostener que cuando participamos en una discusión moral lealmente, tácitamente admitimos que es deseable que nuestro interlocutor (para cualquiera que lo fuese) elija libremente (o aún que actúe en base a) cualquier principio elegido libremente. Pero este presupuesto es muy difícil de justificar.

El análisis de este tema exigiría un estudio cuidadoso de la discusión moral para determinar con exactitud sus presupuestos. El estudio es necesario aún para fundamentar el presupuesto mínimo del discurso moral que manejamos hasta ahora. Pero es una investigación extensa que no puedo encarar en este artículo.

Sinceramente no creo que el hecho de discutir moralmente presupone ningún principio moral, ni aún la existencia de principios morales. Creo que participar lealmente en una discusión moral no es incompatible con una firme creencia en el principio de que si alguien después de reflexión suficiente, llega a un principio moral inválido, debe evitarse coactivamente que lo aplique. Tampoco es incompatible con la creencia de que es valioso que la gente actúe según los principios que juzgue válidos. Ni con la creencia de que las acciones no son algo susceptible de ser moralmente valorado. Quizá haya ciertos rasgos formales que deben satisfacer los principios morales si han de ser postulados como tales. Pero será una forma vacía. Deberá buscarse un método distinto del de la contradicción pragmática, y el uso de los presupuestos del discurso moral. Pero esto, claro, no lo he probado todavía. 